\title{
Dynamic Upstream Wavelength Allocation Method for Power Saving in NG-EPON
}

\author{
Man Soo Han1)
}

\begin{abstract}
In this paper, we propose a novel dynamic wavelength allocation method to save power of the next-generation Ethernet passive optical network (NG-EPON) system. We compare the unused bandwidth in the previous service cycle with the bandwidth of the upstream wavelengths to decide the number of the active upstream wavelengths to be used in the next service cycle. If the unused bandwidth is greater than the predetermined threshold, the number of the active upstream wavelengths is decreased. Otherwise, the number of the active upstream wavelengths is increased. Using a computer simulation to validate the proposed method, we compare the performance of the proposed method with the performance of a static system, where the number of the active upstream wavelengths is fixed. We show performance of the proposed method is comparable to that of the static system. Additionally, we show that the average number of active upstream wavelengths is decreased in the proposed method.
\end{abstract}

Keywords : Dynamic Bandwidth Allocation, Dynamic Wavelength Allocation, EPON, NG-EPON, Performance Evaluation

\section{Introduction}

An NG-EPON is a next-generation EPON technology that supports an upstream transmission speed of $100 \mathrm{Gbps}$ and a downstream transmission speed of $100 \mathrm{Gbps}$ [1]. The NG-EPON system is comprised of one optical line termination (OLT) and many optical network units (ONUs). The NG-EPON uses a broadcasting method to transmit a packet from the OLT to the ONUs. The downstream transmission means the transmission from the OLT to an ONU. The ONU checks the destination of the packet transmitted from the OLT. The packet is accepted by the ONU only if the packet destination is the ONU. The packet is discarded by the ONU if the packet destination is not the ONU. When a packet is transmitted from an ONU to the OLT, only one ONU can transmit a packet to the OLT at a time. If more than or equal to two

Received(March 18, 2019), Review Result(1st: April 04, 2019, 2nd: May 14, 2019), Accepted(June 10, 2019)

1) (Professor) 58554 Dept. Information and Communications Eng., Mokpo National Univ., Muan-gun, Jeonnam, Korea email: mshan@mokpo.ac.kr 
ONUs transmit packets to the OLT simultaneously, a transmission collision occurs. The upstream transmission is the transmission from an ONU to the OLT.

To prevent the collision in the upstream transmission, the OLT collects a report message from an ONU, and then performs a dynamic bandwidth allocation (DBA) operation. During the DBA operation, the OLT assigns a transmission time slot to an ONU. The transmission time slots are not overlapped with each other. The OLT sends the transmission time slot information to an ONU.

Unlike in the EPON system, in the NG-EPON system, multiple upstream wavelengths can be used. For instance, an ONU having an upstream speed of 50 Gbps can use two upstream wavelengths each with a speed of 25 Gbps. Moreover, an ONU having an upstream speed of 100 Gbps can use four upstream wavelengths each with a speed of 25 Gbps.

The power consumption percentage of access networks accounts for $80 \%$ of the total Internet power consumption. It is important to reduce the power consumption of EPON systems, which are the main equipment of access networks. In the NG-EPON system, power can be saved if the number of active upstream wavelengths (AUWs) is reduced in data transmission. However, the performance of the NG-EPON system can worsen if the number of AUWs is reduced. When we decrease the number of AUWs, we have to consider the effect not only on the power saving, but also on the system performance.

Most researches of EPON systems are focused on the performance improvement or on the quality of service (QoS) provision. The dynamic wavelength allocation problem for power saving of EPON systems was introduced in [2]. The number of AUWs is computed by the fraction of the total request size and the wavelength bandwidth in [2]. To provide QoS, a service class must be supported in EPON systems. However, the service class is not considered in the determination of the number of AUWs in [2].

The dynamic wavelength allocation problem for power saving of time and wavelength division multiplexing (TWDM) PON systems was introduced in [3]. However, in [3], an ONU can use any upstream wavelength, whereas in NG-EPON systems, the upstream wavelengths used by ONUs are specified. For instance, if the number of the upstream wavelengths of an ONU is 1, the ONU can use a single arbitrary wavelength among multiple wavelengths and also the ONU can change the wavelength in [3]. As for NG-EPON systems, an ONU can use a fixed single wavelength in the same case. The methods of [3] cannot be directly applied to NG-EPON systems.

In this paper, we propose a novel dynamic wavelength allocation algorithm for upstream data transmission in NG-EPON systems with multiple service classes. The proposed method is 
compliant to the NG-EPON technology standard[4][5] and controls the number of AUWs based on the input traffic load condition. To provide QoS and to minimize the performance degradation, but to save power consumption, the proposed method only reduces the number of AUWs at light input traffic load. Finally, the performance of the proposed algorithm is evaluated by computer simulations.

\section{Dynamic Wavelength Allocation}

The NG-EPON system is comprised of one OLT and N ONUs. An ONU can use multiple upstream wavelengths. The number of the wavelengths of an ONU can be one, two, or four. If an ONU uses multiple wavelengths, the ONU has the same number of upstream transmitters. Therefore, in the NG-EPON system, an ONU cannot change the wavelength of a transmitter. In this study, we assume that each wavelength has an equal transmission speed B. For supporting QoS, three service classes are provided. In each ONU, a queue is maintained for each service class. When a packet arrived at an ONU, the packet is saved to the queue corresponding to the service class of the packet.

The ONU transmits a REPORT message to the OLT, which contains the lengths of the queues in the ONU. The total length of the waiting packets is a transmission request of the ONU. The OLT determines transmission time slots of the ONUs for each upstream wavelength based on the transmission requests of the ONUs. Then the OLT informs the ONU about the transmission time slot using a GATE message. The ONU sends packets to the OLT on the time slot of the GATE message and sends the REPORT message after the data transmission.

Now, we explain the DBA operation that determines the transmission time slots of ONUs. Let $\mathrm{A}(\mathrm{i})$ be the maximum service byte of ONU i. C(ij) is the maximum service byte of class $\mathrm{j}$ of ONU i. In addition, assume that $R(i j)$ is a transmission request of class $j$ of ONU $i$ where $i$ $=1,2, \ldots, N$, and $j=1,2,3$. The maximum total service byte of service class $j, S(j)$, is given by

$$
S(j)=m \sum_{i=1}^{N} C(i j)
$$

where $\mathrm{m}$ is a positive integer. When all ONUs send the REPORT message $\mathrm{m}$ times, the parameter $S(j)$ is recharged by Eq. (1). Let $G(i j)$ be the transmission grant of class $j$ of ONU $i$. Then $\mathrm{G}(\mathrm{ij})$ is calculated by

$$
G(i j)=\min \{\mathrm{A}(\mathrm{i}), \mathrm{S}(\mathrm{j}), \mathrm{R}(\mathrm{ij})\} .
$$


Once $G(i j)$ is obtained, the parameters $A(i), S(j)$, and $R(i j)$ are updated by $A(i)=A(i)-G(i j)$, $S(j)=S(j)-G(i j)$, and $R(i j)=R(i j)-G(i j)$, respectively. The parameter $A(i)$ is recharged when the DBA operation for ONU i starts. The DBA operation for service class 1 is performed first, then for service class 2, and finally for service class 3 . Figure 1 shows the pseudo code of the DBA operation for class $\mathrm{j}$ of ONU $\mathrm{i}$.

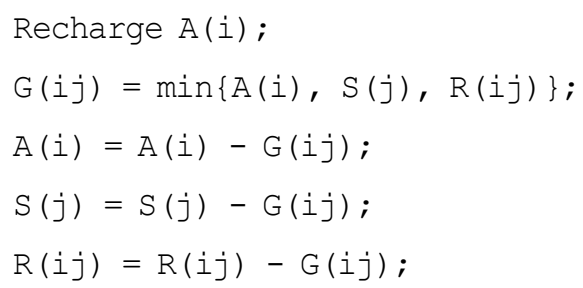

[Fig. 1] Pseudo Code of DBA Operation

When the DBA operation is finished, then the grant $G(i j)$ is allocated to the upstream wavelengths. The fixed partial amount of the grant $\mathrm{G}(\mathrm{ij})$ is added to the allocation amount of the least used wavelength. The grant $\mathrm{G}(\mathrm{ij})$ is then decreased by the fixed amount. The used amount of the least used wavelength is increased by the fixed amount. Then we recalculate the least used wavelength and repeat the $G(\mathrm{ij})$ allocation procedure until the grant $G(\mathrm{ij})$ is fully allocated.

Now, we explain the dynamic upstream wavelength allocation method. Assume w(i) is the number of the AUWs of ONU i. In this study, $w(i)=1,2$, or 4 . If ONU $i$ uses only one upstream wavelength, we cannot dynamically determine the number of AUWs since the ONU needs at least one upstream wavelength. If ONU i uses more than one upstream wavelength, we can dynamically determine the number of AUWs by considering the input traffic load. In this study, the number of AUWs is decreased if the input traffic load is light. Otherwise, the number of AUWs is increased.

First, we define a service cycle for the dynamic upstream wavelength allocation. A service cycle is defined by the time when all ONUs receive a transmission service once. Each ONU has to receive a transmission service to transmit packets or to transmit a REPORT message to the OLT. Therefore, each ONU receives a transmission service only once per one service cycle. To determine the number of AUWs, we check the unused bandwidth of all ONUs in the previous service cycle. Then we compare the unused bandwidth with the bandwidth sum of the upstream wavelengths. 
Let $\mathrm{v}_{1}(\mathrm{i})$ be the variable that denotes the maximum number of the wavelengths of ONU $i$, which is exactly 1 . The variable $\mathrm{v}_{1}(\mathrm{i})$ is 1 if $\mathrm{w}(\mathrm{i})=1$; otherwise, $\mathrm{v}_{1}(\mathrm{i})=0$. Let $\mathrm{v}_{2}(\mathrm{i})$ be the variable that denotes the maximum number of the wavelengths of ONU i, which is exactly 2. The variable $\mathrm{v}_{2}(\mathrm{i})$ is 1 if $\mathrm{w}(\mathrm{i})=2$; otherwise, $\mathrm{v}_{2}(\mathrm{i})=0$. Let $\mathrm{v}_{4}(\mathrm{i})$ be the variable that denotes the maximum number of the wavelengths of ONU $i$, which is exactly 4 . Similarly, the variable $v_{4}(i)$ is 1 if $\mathrm{w}(\mathrm{i})=4$; otherwise, $\mathrm{v}_{4}(\mathrm{i})=0$. Let $\mathrm{W}_{1}$ and $\mathrm{W}_{2}$ be the numbers of ONUs that use exactly one upstream wavelength and two upstream wavelengths, respectively. In addition, suppose that $W_{4}$ is the number of ONUs that use exactly four upstream wavelengths. Then we have

$$
\begin{aligned}
& W_{1}=\sum_{i=1}^{N} \mathrm{v}_{1}(\mathrm{i}), \\
& W_{2}=\sum_{i=1}^{N} \mathrm{v}_{2}(\mathrm{i}), \\
& W_{4}=\sum_{i=1}^{N} \mathrm{v}_{4}(\mathrm{i}) .
\end{aligned}
$$

The maximum upstream bandwidth is $\mathrm{B}\left(\mathrm{W}_{1}+2 \mathrm{~W}_{2}+4 \mathrm{~W}_{4}\right)$. Let $\mathrm{E}$ be the sum of unused service bytes of all ONUs in the previous service cycle. In other words, E is the sum of unused remainder of $\mathrm{A}(\mathrm{i})$ at the previous service cycle. That is

$$
E=\sum_{i=1}^{N} \mathrm{~A}(\mathrm{i}) \text {. }
$$

If $\mathrm{E}=2 \mathrm{~B} \cdot \mathrm{W}_{4}$, then two of the four upstream wavelengths were used in average in the previous service cycle. Therefore, if $\mathrm{E}>2 \mathrm{~B} \cdot \mathrm{W}_{4}$, this indicates that $\mathrm{ONU} \mathrm{i}$, having $\mathrm{w}(\mathrm{i})=4$, needs to use only two active wavelengths in the next service cycle. If $E=B \cdot W_{2}+3 B \cdot W_{4}$, then one upstream wavelength was used in average in the previous service cycle. Therefore, if $\mathrm{E}>$ $\mathrm{B} \cdot \mathrm{W}_{2}+3 \mathrm{~B} \cdot \mathrm{W}_{4}$, then ONU $\mathrm{i}$, having $\mathrm{w}(\mathrm{i})=2$ or $\mathrm{w}(\mathrm{i})=4$, needs to use only a single active wavelength in the next service cycle. Figure 2 summarizes the dynamic wavelength allocation process.

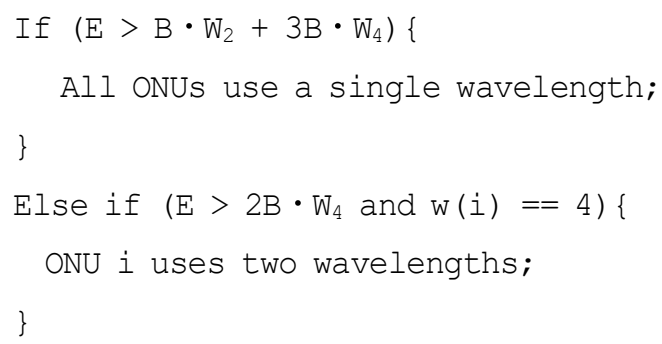

[Fig. 2] Pseudo Code of Dynamic Wavelength Allocation Operation 


\section{Performance Evaluation}

For performance evaluation, we consider an NG-EPON system which has 16 ONUs, i.e., $\mathrm{N}=$ 16 as in [6]. The transmission speed of the upstream wavelengths is B = 10 Gbps. We assume that the distance between the OLT and the ONUs is given by $20 \mathrm{Km}$. In addition, each ONU has three service classes, and the queue size is 4 Mbytes for each service class. We suppose that ONUs $1-6$ have $\mathrm{w}(\mathrm{i})=1$, ONUs $7-12$ have $\mathrm{w}(\mathrm{i})=2$, and ONUs $13-16$ have $\mathrm{w}(\mathrm{i})=4$. The maximum input traffic speeds of ONUs with $\mathrm{w}(\mathrm{i})=1,2$, and 4 are 1, 2.6, and 10.6 Gbps, respectively.

In this study, we use the self-similar traffic model that the traffic is generated by a number of Pareto-distributed on-off traffic processes [6]. The shape parameters of the on and off intervals are 1.4 and 1.2, respectively. The packet size follows the tri-modal distribution where the packet sizes are 64, 500, and 1500 bytes and their load fractions are 60, 20, and 20\%, respectively, as in [6]. Also we assume that the traffic loads are balanced among the traffic classes. This means that the service class of an input packet in an ONU is determined with the same probability, $1 / 3$.

For comparison, we introduce a static NG-EPON system, in which the number of AUWs is fixed in each ONU. Therefore, the number of AUWs of ONU i, w(i), is constant in the static system. The performance of the static system is always better than that of the proposed method, but the power saving is worse. By increasing the input traffic load rates of all ONUs from 0.1 to 0.99 , we simulate each system. The simulation is executed until the total number of packets transmitted by ONUs exceeds $10^{9}$ for each system.

Figures 3 and 4 show the mean delays of service classes 1, 2, and 3. In Figures 3 and 4, performance of the proposed method is worse than that of the static system in mean delay. Since the static system always uses the maximum number of wavelengths under any traffic conditions, its performance is better than that of the proposed method. However, the static system uses more power than the proposed method. Figure 5 illustrates the average number of AUWs for the proposed method. 


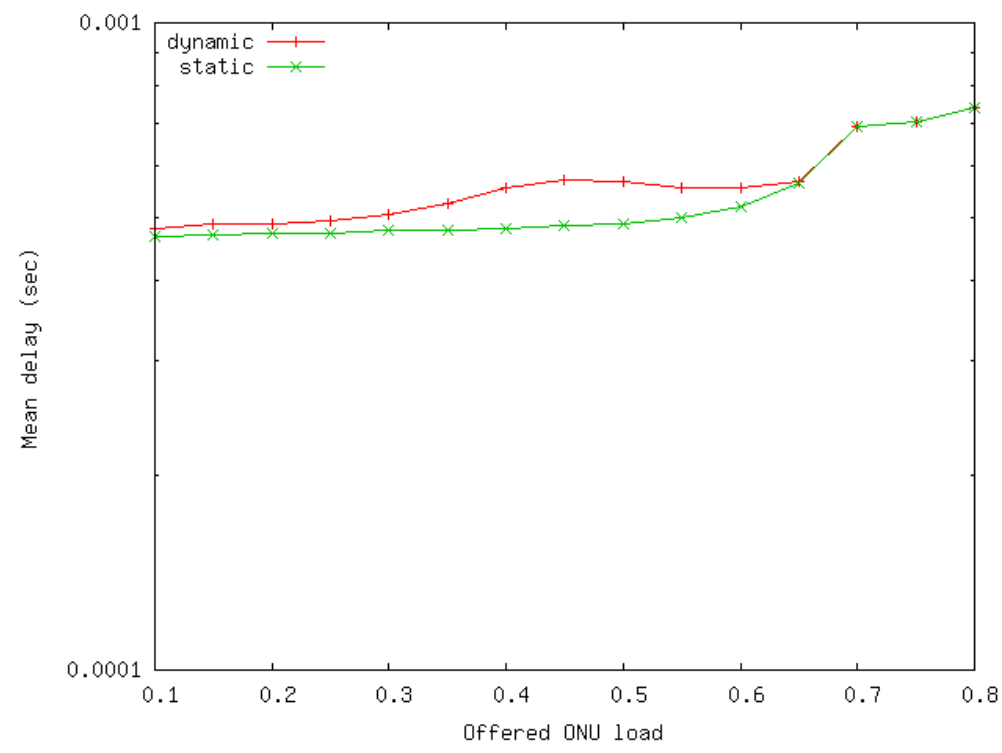

[Fig. 3] Mean Delay of Service Class 1

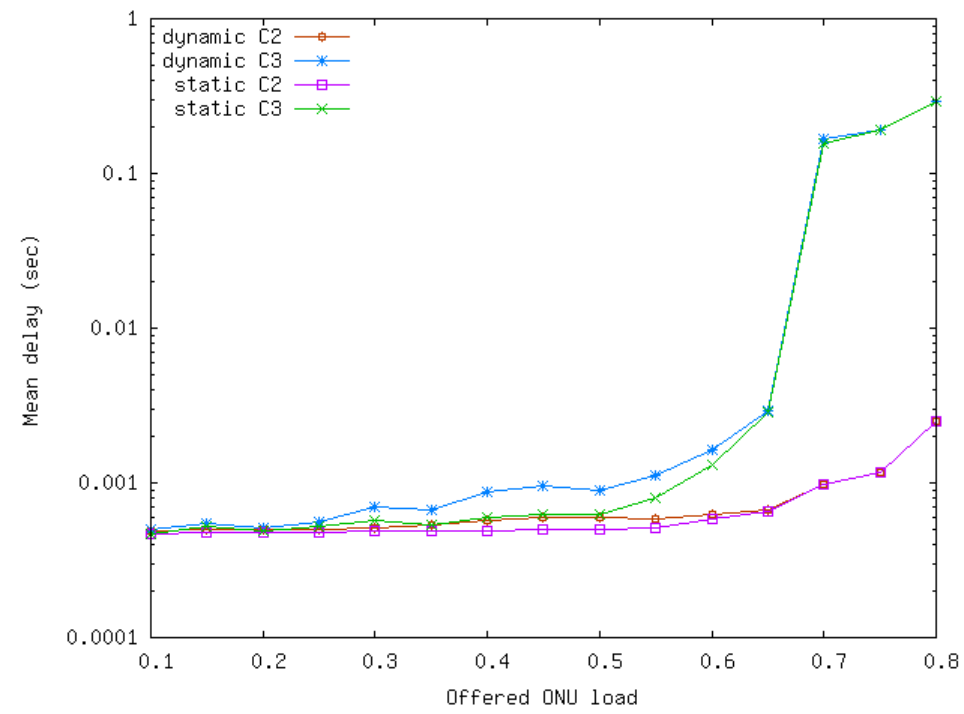

[Fig. 4] Mean Delay of Service Classes 2 and 3 


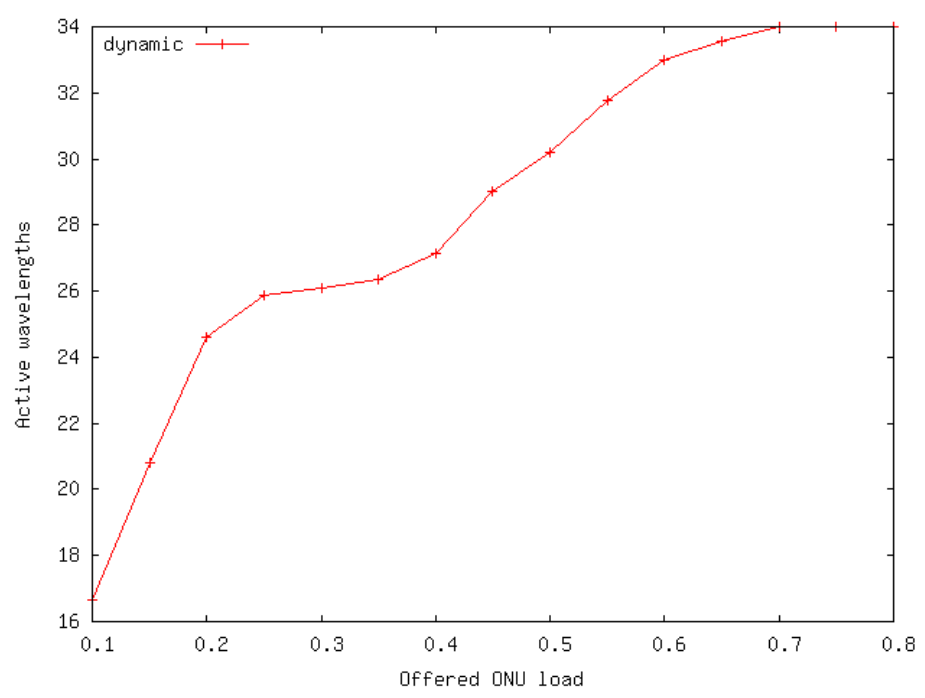

[Fig. 5] Average Number of Active Wavelengths

\section{Conclusions}

We propose a novel dynamic wavelength allocation method for power saving of the NG-EPON system. We check the unused bandwidth in the previous service cycle and then compare it with the bandwidth of the upstream wavelengths. If the unused bandwidth is greater than the predetermined threshold, the number of active wavelengths is decreased in the upcoming service cycle. Using a simulation, we showed that the performance of the proposed method is comparable to that of the static system, in which the number of active wavelengths is fixed. Additionally, we showed that the average number of active upstream wavelengths is decreased in the proposed method.

\section{References}

[1] G. Kramer, Flexible and Extensible Architecture for Multiple Generations of NG-EPON, www.ieee802.org/3/ca/public/meeting_archive/2016/03/kramer_3ca_1a_0316.pdf, Mar 1 (2016)

[2] A. Dixit, B. Lannoo, D. Colle, M. Pickavert, P. Demeester, Novel DBA algorithm for Energy Efficiency in TWDM-PONs, 39th European Conference and Exhibition on Optical Communication (ECOC), (2013), September 22-26; London, UK

[3] M. S. Han, Energy Efficient Dynamic Wavelength and Bandwidth Allocation for TWDM PON, 
Contemporary Engineering Sciences, (2014), Vol.7, No.23, pp.1303-1311.

[4] G. Kramer, MPCP+: A proposal for Channel Bonding at MAC Control Sublayer, www.ieee802.org/3/ca/public/meeting_archive/2016/03/kramer_3ca_2a_0316.pdf, Mar 1 (2016)

[5] G. Kramer, Channel Bonding in Reconciliation Sublayer (Upstream Direction), www.ieee802.org/3/ca/public/meeting_archive/2016/07/kramer_3ca_3c_0716.pdf, Jul 1 (2016)

[6] M. S. Han, H. Yoo, and D. S. Lee, Development of Efficient Dynamic Bandwidth Allocation Algorithm for XGPON, ETRI Journal, (2013), Vol.35, No.1, pp.18-26. 